\title{
TWO PARALLEL SINGLE PHASE RECTIFIERS BY USING SINGLE PHASE TO THREE PHASE INDUCTION MOTOR
}

\author{
Vaddi Ramesh $^{1}$, P Anjappa ${ }^{2}$, P.Dhanamjaya ${ }^{3}$ \\ ${ }^{1}$ PH.D Student, Department of Electrical and Electronics Engineering, KL University, Guntur (AP) India) \\ ${ }^{2} \mathrm{HOD} \&$ Associate professor, ${ }^{3}$ Assistant professor, Department of EEE, Golden valley integrated campus, Madanapalli, \\ A.P, India,rameshvaddi6013@gmail.com,anji_abhi@yahoo.co.in,dhanam302@gmail.com
}

\begin{abstract}
A single-phase to three-phase drive system composed of two parallel single-phase rectifiers, a three-phase inverter and an induction motor was proposed. The system combines two parallel rectifiers without the use of transformers. The system model and the control strategy, including the PWM technique, have been developed.

The complete comparison between the proposed and standard configurations has been carried out in this paper. Compared to the conventional topology, the proposed system permits to reduce the rectifier switch currents, the THD of the grid current with same switching frequency or the switching frequency with same THD of the grid current and to increase the fault tolerance characteristics. In addition, the losses of the proposed system may be lower than that of the conventional counterpart.

The initial investment of the proposed system (due to high number of semiconductor devices) cannot be considered a drawback, especially considering the scenario where the cited advantages justify such initial investment. The experimental results have shown that the system is controlled properly, even with transient and occurrence of fault
\end{abstract}

\section{INTRODUCTION}

Several solutions have been proposed when the objective is to supply a three-phase motor from single-phase ac mains. It is quite common to have only a single phase power grid in residential, commercial, manufacturing, and mainly in rural areas, while the adjustable speed drives may request a threephase power grid. Single-phase to three-phase ac-dc-ac conversion usually employs a full-bridge topology, which implies in ten power switches. This converter is denoted here as conventional topology. Parallel converters have been used to improve the power capability, reliability, efficiency, and redundancy. Parallel converter techniques can be employed to improve the performance of active power filters, uninterruptible power supplies (UPS), fault tolerance of doubly fed induction generators, and three-phase drives. Usually the operation of converters in parallel requires a transformer for isolation. However, weight, size, and cost associated with the transformer may make such a solution undesirable. When an isolation transformer is not used, the reduction of circulating currents among different converter stages is an important objective in the system design.

In this paper, a single-phase to three-phase drive system composed of two parallel single-phase rectifiers and a threephase inverter is proposed. The proposed system is conceived to operate where the single-phase utility grid is the unique option available. Compared to the conventional topology, the proposed system permits to reduce the rectifier switch currents, the total harmonic distortion (THD) of the grid current with same switching frequency or the switching frequency with same THD of the grid current, and to increase the fault tolerance characteristics. In addition, the losses of the proposed system may be lower than that of the conventional counterpart. The aforementioned benefits justify the initial investment of the proposed system, due to the increase of number of switches.

\section{COMPONENTS INPROPOSED CIRCUIT}

\subsection{Effect of Source Inductance}

In the Fig. $1,1 g, 1_{a}, l_{b}, l_{b}$, are input side inductors these inductors are called source inductors. The input side bridge rectifier is a fully controlled rectifier. The presence of source inductance introduces an additional mode of operation of when firing angle is less than certain value.

When there is an inductor in series with each input line, it is necessary to find out its effect. The effects are

1. The reduction in output voltage

2. The duration of commutation overlap.

3. The relationship between the firing angle and the commutation overlap. 


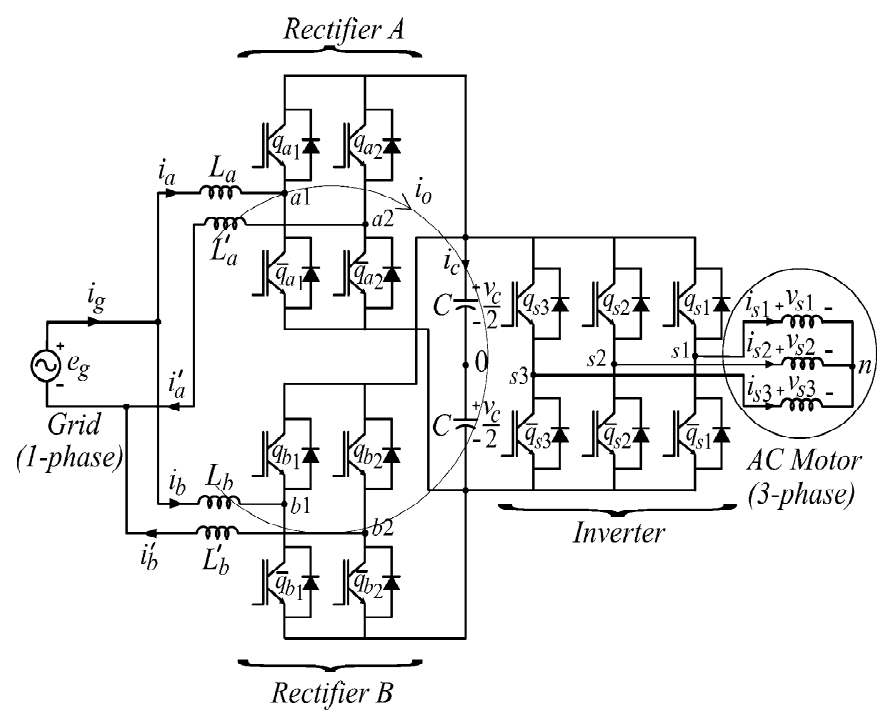

Fig 1 Proposed single-phase to three-phase drive system

\subsection{Link Capacitor}

A simple analytical expression for the current stress on the DC-link capacitor caused by the load-side inverter of a voltage DC-link-converter system is derived. The DC-link capacitor current RMS value is determined from the modulation depth and by the amplitude and the phase angle of the inverter output current assuming a sinusoidal inverter output current and a constant DC-link voltage.

Despite neglecting the output-current ripple, the results of the analytical calculation are within $8 \%$ of measurements made from digital simulation and an experimental system, even if the output-current ripple is relatively high as in the case of low-frequency IGBT inverter systems. The simple analytical expression provides significant advantages over simulation methods for designing the DC-link capacitor of PWM converter systems.

\subsection{IGBT Fundamentals}

The Insulated Gate Bipolar Transistor (IGBT) is a minoritycarrier device with high input impedance and large bipolar current-carrying capability. Many designers view IGBT as a device with MOS input characteristics and bipolar output characteristic that is a voltage-controlled bipolar device. To make use of the advantages of both Power MOSFET and BJT, the IGBT has been introduced. It's a functional integration of Power MOSFET and BJT devices in monolithic form. It combines the best attributes of both to achieve optimal device characteristics.

The IGBT is suitable for many applications in power electronics, especially in Pulse Width Modulated (PWM) servo and three-phase drives requiring high dynamic range control and low noise. It also can be used in Uninterruptible Power Supplies (UPS), Switched-Mode Power Supplies (SMPS), and other power circuits requiring high switch repetition rates. IGBT improves dynamic performance and efficiency and reduced the level of audible noise. It is equally suitable in resonant-mode converter circuits. Optimized IGBT is available for both low conduction loss and low switching loss.

SCR, the Triac conducts in either direction when turned on. The Triac also differs from the SCR in that either a positive or negative gate signal triggers it into conduction. Thus the Triac is a three terminal, four layer bidirectional semiconductor device that controls ac power whereas an SCR controls dc power or forward biased half cycles of ac in a load. Because of its bidirectional conduction property, the Triac is widely used in the field of power electronics for

It is wound for a definite number of poles. The windings are geometrically spaced 120 degrees apart. Two types of rotors are used in Induction motors Squirrel cage rotor and Wound rotor.

\section{PULSE WIDTHMODULATION}

\subsection{General Description}

The PWM component provides compare outputs to generate single or continuous timing and control signals in hardware. The PWM is designed to provide an easy method of generating complex real-time events accurately with minimal CPU intervention. PWM features may be combined with other analog and digital components to create custom peripherals. The PWM generates up to two left- or right-aligned PWM outputs or one centered-aligned or dual edged PWM output. The PWM outputs are double buffered to avoid glitches caused by duty cycle changes while running. Left-aligned PWMs are used for most general-purpose PWM uses .Rightaligned PWMs are typically only used in special cases that require alignment opposite of left-aligned PWMs. Dual-edged PWMs are optimized for power conversion where phase alignment must be adjusted.

The optional dead band provides complementary outputs with adjustable dead time where both outputs are low between each transition. The complementary outputs and dead time are most often used to drive power devices in half-bridge configurations to avoid shoot-through current sand resulting damage. A kill input is also available that immediately disables the dead band outputs when enabled. Three kill modes are available to support multiple use scenarios. Two hardware dither modes are provided to increase PWM flexibility. The first dither mo de increases effective resolution by two bits when resources or clock frequency preclude a standard implementation in the PWM counter. 
The second dither mode uses a digital input to select one of the two PWM outputs on a cycle-by-cycle basis, this mode is typically used to provide fast transient response in power converts. The trigger and reset inputs allow the PWM to be synchronized with other internal or external hardware. The optional trigger input is configurable so that a rising edge starts the PWM. A rising edge on the reset input causes the PWM counter to reset its count as if the terminal count was reached. The enable input provides hardware enable to gate PWM operation based on a hardware signal. An interrupt can be programmed to be generated under any combination of the following conditions. When the PWM reaches the terminal count or when a compare output goes high.

The most common use of the PWM is to generate periodic waveforms with adjustable duty cycles. The PWM also provides optimized features for power control, motor control, switching regulators, and lighting control. You can also use the PWM as a clock divider by driving a clock into the clock input and using the terminal count or a PWM output as the divided clock output. PWMs, timers, and counters share many capabilities, but each provides specific capabilities. A Counter component is better used in situations that require the counting of a number of events but also provides rising edge capture input as well as a compare output.

\subsection{Space Vector PWM}

The initial use of Space Vector Modulation at three-phase voltage-source inverters has been expanded by application to novel three-phase topologies as AC/DC Voltage Source Converter, AC/DC or DC/AC Current Source Converters, Resonant Three-Phase Converters, B4-inverter, Multilevel Converters, AC/AC Matrix Converters, and so on. The Space Vector PWM generation module accepts modulation index commands and generates the appropriate gate drive waveforms for each PWM cycle. This section describes the operation and configuration of the SVPWM module.

A three-phase 2-level inverter with dc link configuration can have eight possible switching states, which generates output voltage of the inverter. Each inverter switching state generates a voltage Space Vector (V1 to V6 active vectors, V7 and V8 zero voltage vectors) in the Space Vector plane. The magnitude of each active vector (V1to V6) is $2 / 3 \mathrm{Vdc}$ (dc bus voltage).

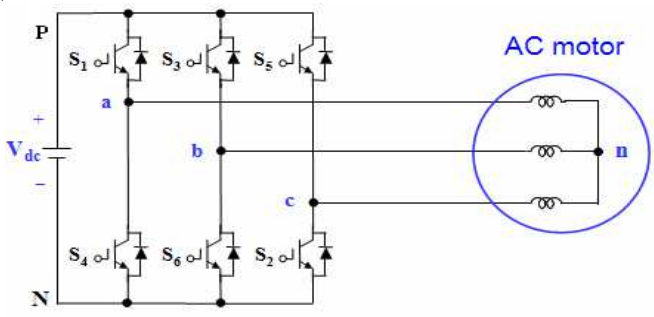

Fig 2 A three-phase two-level inverter with dc link configuration
The three-phase inverter presented in Fig 2 is here in considered. Fig 3 presents the appropriate output voltages without PWM (six-step).

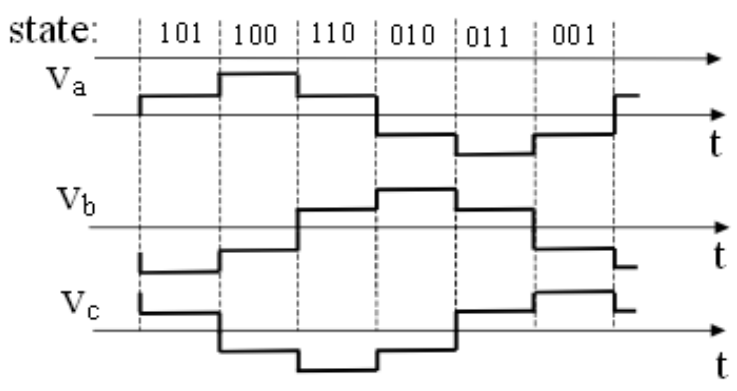

Fig 3 Output voltage waveforms and state coding for the case Without PWM

Each state of the output voltage system leads to a switching vector in the complex plane. It results 6 active switching vectors $\mathrm{V} 1 \ldots \mathrm{V} 6$ and 2 vectors corresponding to the zero states. The magnitude of the active vectors is $(2 / 3) \mathrm{Vdc}$. Since the output voltages are at $2 \mathrm{pi} / 3$ out of phase each other, the Space Vectors system can occupy a number of positions with an order multiple of three.

\subsection{Principle of Pulse Width Modulation (PWM)}

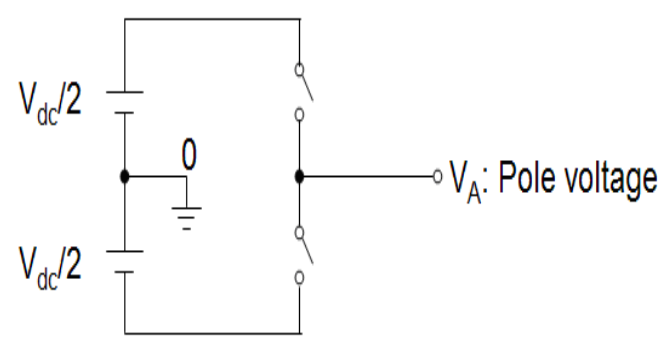

Fig 4 Circuit model of a single-phase inverter with a centretaped grounded DC bus
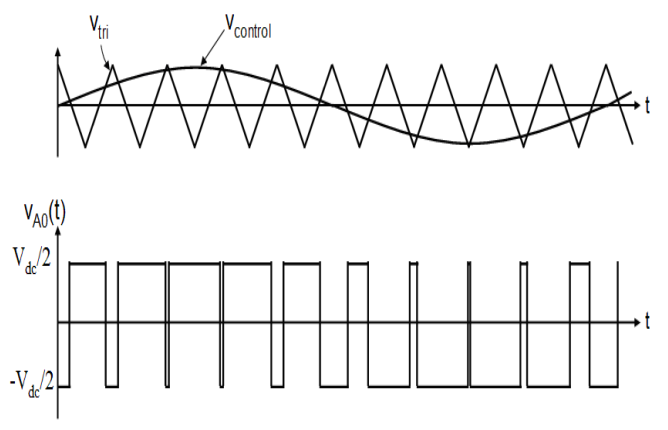

Fig 5 Pulse width modulation 
As depicted in Fig 4 the inverter output voltage is determined in the following

When $\mathrm{V}$ control $>\mathrm{V}$ tri, $\mathrm{V}_{\mathrm{A} 0}=\mathrm{V}$ dc $/ 2$

When $\mathrm{V}$ control $<\mathrm{V}$ tri, $\mathrm{V}_{\mathrm{A} 0}=\mathrm{V} \mathrm{dc} / 2$

Also, the inverter output voltage has the following features PWM frequency is the same as the frequency of $\mathrm{V}$ tri Amplitude is controlled by the peak value of $\mathrm{V}$ control Fundamental frequency is controlled by the frequency of $\mathrm{V}$ control

\section{RECTIFIER AND INVERTER}

\subsection{AC to DC converter}

A rectifier is an electrical device that converts alternating current (AC), which periodically reverses direction, to direct current (DC), which is in only one direction, a process known as rectification. Rectifiers have many uses including as components of power supplies and as detectors of radio signals. Rectifiers may be made of solid state diodes, vacuum tube diodes, mercury arc valves, and other components.

When only one diode is used to rectify AC (by blocking the negative or positive portion of the waveform), the difference between the term diode and the term rectifier merely one of usage, i.e, the term rectifier is describes a diode that is being used to convert AC to DC.

\subsubsection{Half-Wave Rectification}

In half wave rectification, either the positive or negative half of the AC wave is passed, while the other half is blocked. Because only one half of the input waveform reaches the output, it is very inefficient if used for power transfer. Halfwave rectification can be achieved with a single diode in a one-phase supply, or with three diodes in a three-phase supply.
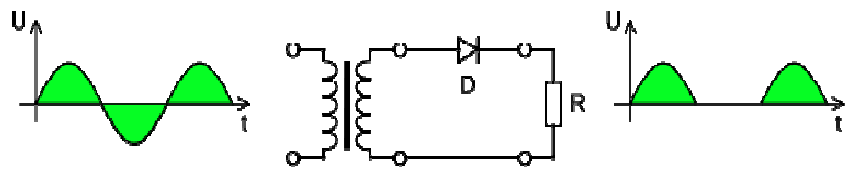

Fig 6 Half wave rectification

The output DC voltage of a half wave rectifier can be calculated with the following two ideal equations

$$
\begin{gathered}
V_{\text {rms }}=\frac{V_{\text {peak }}}{2} \\
V_{\text {dc }}=\frac{V_{\text {peak }}}{\pi}
\end{gathered}
$$

\subsubsection{Full-Wave Rectification}

A full-wave rectifier converts the whole of the input waveform to one of constant polarity (positive or negative) at its output.
Full-wave rectification converts both polarities of the input waveform to DC (direct current), and is more efficient. However, in a circuit with a non-centre transformer, four diodes are required instead of the one needed for half-wave rectification. Four diodes arranged this way are called a diode bridge or bridge rectifier.

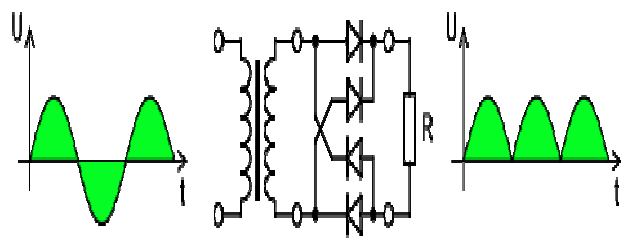

Fig 7 A Full-Wave Rectifier Using Four Diodes

For single-phase AC, if the transformer is centre-tapped, then two diodes back-to-back (i.e. anodes-to-anode or cathode-tocathode) can form a full-wave rectifier. Twice as many windings are required on the transformer secondary to obtain the same output voltage compared to the bridge rectifier above.

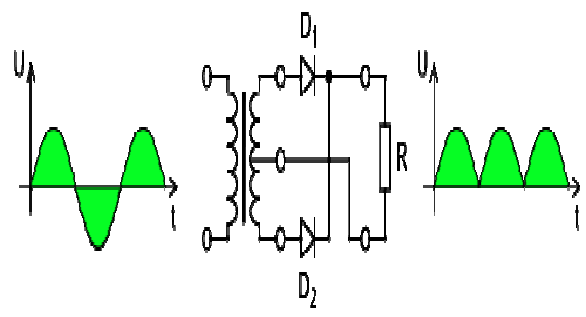

Fig 8 Full-wave rectifier using a centre tap transformer and two diodes

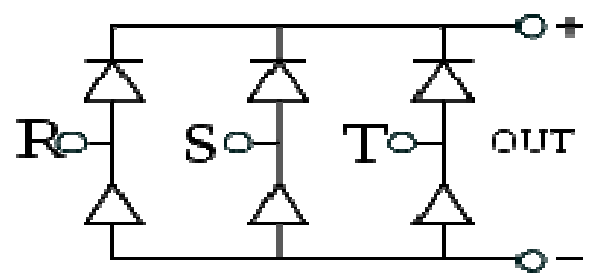

Fig 9 A three-phase bridge rectifier

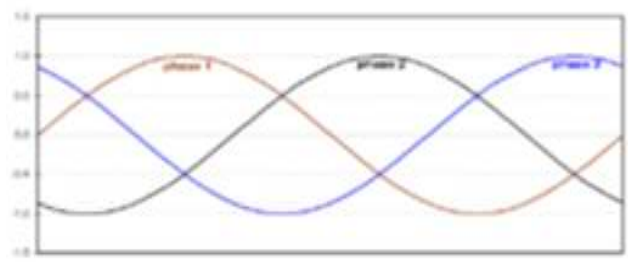

Three phase AC input 


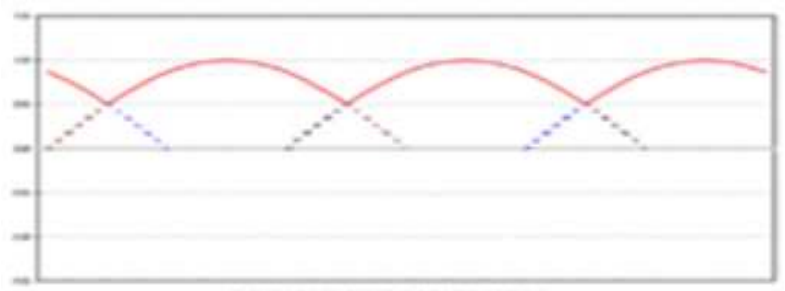

Three phase half wave rectifier

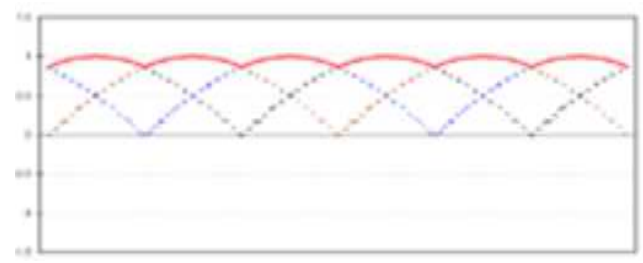

Three phase full wave rectifier

Fig 10 Three-phase AC input, half and full wave rectified DC output waveforms

For three-phase AC, six diodes are used. Typically there are three pairs of diodes, each pair, though, is not the same kind of double diode that would be used for a full wave singlephase rectifier. Instead the pairs are in series (anode to cathode). Typically, commercially available double diodes have four terminals so the user can configure them as singlephase split supply use, for half a bridge, or for three-phase use.Most devices that generate alternating current (such devices are called alternators) generate three-phase AC. For example, an automobile alternator has six diodes inside it to function as a full-wave rectifier for battery charging applications. The average and root-mean-square output voltages of an ideal single phase full wave rectifier can be calculated as

$$
\begin{gathered}
\mathrm{V}_{\mathrm{dc}}=\mathrm{V}_{\mathrm{av}}=\frac{2 \mathrm{~V}_{\mathrm{p}}}{\pi} \\
\mathrm{V}_{\mathrm{rms}}=\frac{\mathrm{V}_{\mathrm{p}}}{\sqrt{2}}
\end{gathered}
$$

Where

$\mathrm{V}_{\mathrm{dc}}$ and $\mathrm{V}_{\mathrm{av}}$ are the average and DC output voltage.

$V_{p}$ is the peak value of half wave.

The extreme of this approach is to dispense with the reservoir capacitor altogether and put the rectified waveform straight into a choke-input filter. The advantage of this circuit is that the current waveform is smoother and consequently the rectifier no longer has to deal with the current as a large current pulse, but instead the current ctrical device that converts direct current (DC) to alternating current (AC), the converted $\mathrm{AC}$ can be at any required voltage and frequency with the use of appropriate transformers, switching, and control circuits.

Solid-state inverters have no moving parts and are used in a wide range of applications, from small switching power supplies in computers, to large electric utility high-voltage direct current applications that transport bulk power. Inverters are commonly used to supply AC power from DC sources such as solar panels or batteries. There are two main types of inverter. The output of a modified sine wave inverter is similar to a square wave output except that the output goes to zero volts for a time before switching positive or negative. Thus it is compatible with all AC electronic devices. This is the type used in grid-tie inverters. Its design is more complex, and costs 5 or 10 times more per unit power. The electrical inverter is a high-power electronic oscillator. It is so named because early mechanical AC to DC converters was made to work in reverse, and thus were "inverted", to convert DC to AC. The inverter performs the opposite function of a rectifier.

\section{MODELLING AND CASE STUDY}

\subsection{System Model}

The system is composed of grid, input inductors $\left(L_{a}, L_{a}^{\prime}, L_{b}, L_{b}^{\prime}\right)$. Rectifiers (A and B), capacitor bank at the dc-link, inverter, and induction machine Rectifiers A and B are constituted of switches $q_{a 1}, \bar{q}_{a 1}, q_{a 2}$ and $\bar{q}_{a 2}$ and $q_{b 1}, \bar{q}_{b 1}, q_{b 2}$ and $\bar{q}_{b 2}$ respectively. The inverter is constituted of switches $q_{s 1}, \bar{q}_{s 1}, q_{s 2}, \bar{q}_{s 2}, q_{s 3}$ and $\bar{q}_{s 3}$.

From Fig.12, the following equations can be derived for the front-end rectifier

$$
\begin{aligned}
& v_{a 10}-v_{a 20}=e_{g}-\left(r_{a}+l_{a} p\right) i_{a}-\left(r_{a}^{\prime}+l_{a}^{\prime} p\right) i_{a}^{\prime} \\
& v_{b 10}-v_{b 20}=e_{g}-\left(r_{b}+l_{b} p\right) i_{b}-\left(r_{b}^{\prime}+l_{b}^{\prime} p\right) i_{b}^{\prime} \\
& v_{a 10}-v_{b 10}=\left(r_{b}+l_{b} p\right) i_{b}-\left(r_{a}+l_{a} p\right) i_{a} \\
& v_{a 20}-v_{b 20}=\left(r_{a}^{\prime}+l_{a}^{\prime} p\right) i_{a}^{\prime}-\left(r_{b}^{\prime}+l_{b}^{\prime} p\right) i_{b}^{\prime} \\
& i_{g}=i_{a}+i_{b}=i_{a}^{\prime}+i_{b}^{\prime}
\end{aligned}
$$

Where $p=d / d t$ and symbols like $r$ and $l$ represent the resistances and inductances of the input inductors $L_{a}, L_{a}^{\prime}, L_{b}, L_{b}^{\prime}$.

The circulating current $i_{0}$ can be defined from $i_{a}, i_{a}^{\prime} i_{b}, i_{b}^{\prime}$.

$i_{0}=i_{a}-i_{a}^{\prime}=-i_{b}+i_{b}^{\prime}$

Introducing $i_{0}$ and adding (3) and (4), relations (1)-(4) Become 
$v_{a}=e_{g}-\left[\left(r_{a}+r_{a}^{\prime}\right)+\left(l_{a}+l_{a}^{\prime}\right) p\right] i_{a}+\left(r_{a}^{\prime}+l_{a}^{\prime} p\right) i_{0}$

$v_{b}=e_{g}-\left[\left(r_{b}+r_{b}^{\prime}\right)+\left(l_{b}+l_{b}^{\prime}\right) p\right] i_{b}+\left(r_{b}^{\prime}+l_{b}^{\prime} p\right) i_{0}$

$v_{0}=-\left[\left(r_{a}^{\prime}+r_{b}^{\prime}\right)+\left(l_{b}+l_{b}^{\prime}\right) p\right] i_{0}$

$\left[\left(r_{b}-r_{b}^{\prime}\right)+\left(l_{b}+l_{b}^{\prime}\right) p\right] i_{b}$

$$
-\left[\left(r_{a}-r_{a}^{\prime}\right)+\left(l_{a}-l_{a}^{\prime}\right) p\right] i_{a}+
$$

Where

$v_{a}=v_{a 10}-v_{a 20}$

$v_{b}=v_{b 10}-v_{b 20}$

$v_{a}=v_{a 10}+v_{a 20}-v_{b 10}-v_{b 20}$

Relations (7)-(9) and (5) constitute the front-end rectifier dynamic model. Therefore, $v_{a}$ (rectifier A), $v_{b}$ (rectifier B), and $v_{0}$ (rectifiers A and B) are used to regulate currents $i_{a}, i_{b}, i_{0}$ respectively. Reference currents $i_{a}^{*}$ and $i_{b}^{*}$ are chosen equal to $i_{g}^{*} / 2$ and the reference circulating current $i_{0}^{*}$ is chosen equal to 0 .

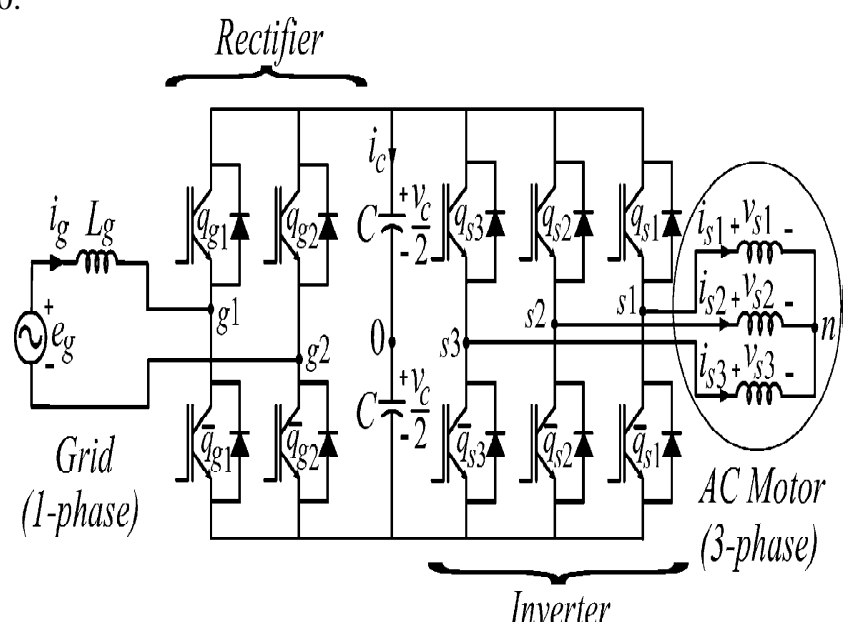

Fig 11 Single-phase to three-phase drive system

In order to both facilitate the control and share equally current, voltage, and power between the rectifiers, the four inductors should be equal, i.e. $r_{g}^{\prime}=r_{a}=r_{a}^{\prime}=r_{b}=r_{b}^{\prime}$ and $l_{g}^{\prime}=l_{a}=$ $l_{a}^{\prime}=l_{b}=l_{b}^{\prime}$. In this case, the model (7)-(9) can be simplified to the model given by

$$
\begin{aligned}
& v_{a}+\frac{v_{0}}{2}=e_{g}-2\left(r_{g}^{\prime}+l_{g}^{\prime} p\right) i_{a} \\
& v_{a}-\frac{v_{0}}{2}=e_{g}-2\left(r_{g}^{\prime}+l_{g}^{\prime} p\right) i_{b} \\
& v_{0}=-2\left(r_{g}^{\prime}+l_{g}^{\prime} p\right) i_{0}
\end{aligned}
$$

Additionally, the equations for $i_{g}, i_{a}^{\prime}$ and $i_{b}^{\prime}$ can be written as

$$
\begin{aligned}
& v_{a b}=\frac{v_{a}+v_{b}}{2}=e_{g}-\left(r_{g}^{\prime}+l_{g}^{\prime} p\right) i_{g} \\
& v_{a}-\frac{v_{0}}{2}=e_{g}-2\left(r_{g}^{\prime}+l_{g}^{\prime} p\right) i_{a}^{\prime} \\
& v_{a}+\frac{v_{0}}{2}=e_{g}-2\left(r_{g}^{\prime}+l_{g}^{\prime} p\right) i_{b}^{\prime}
\end{aligned}
$$

In this ideal case (four identical inductors), the circulating current can be reduced to zero imposing.

$$
v_{0}=v_{a 10}+v_{a 20}-v_{b 10}-v_{b 20}=0
$$

When $i_{0}=0\left(i_{a}=i_{a}^{\prime}, i_{b}=i_{b}^{\prime}\right)$ the system model (7)-(9) is reduced to

$$
\begin{aligned}
& v_{a}=e_{g}-2\left(r_{g}^{\prime}+l_{g}^{\prime} p\right) i_{a} \\
& v_{b}=e_{g}-2\left(r_{g}^{\prime}+l_{g}^{\prime} p\right) i_{b}
\end{aligned}
$$

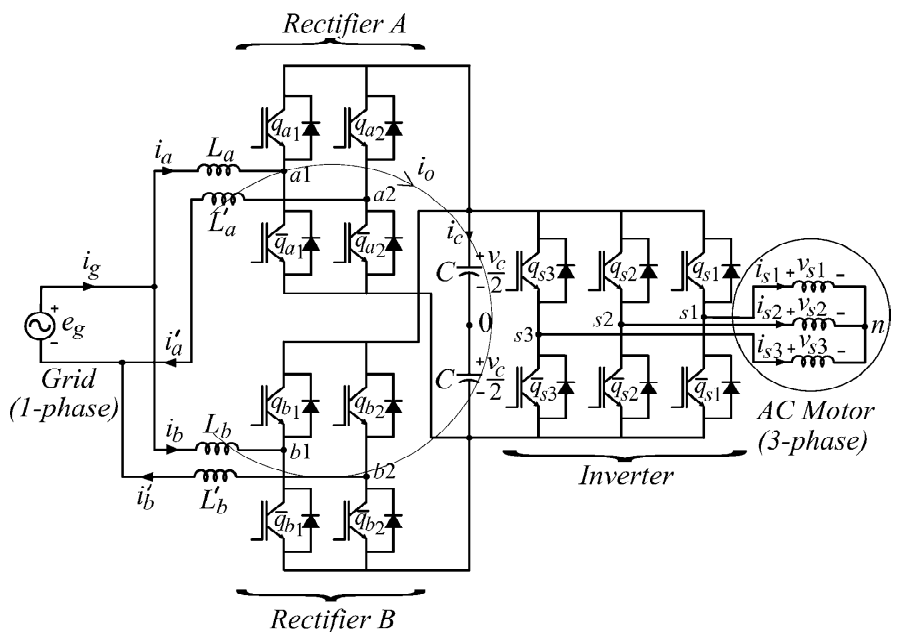

Fig 12 Proposed single-phase to three-phase drive system

\subsection{PWM Strategy}

The PWM strategy for the rectifier will be presented. The rectifier pole voltages $v_{a 10}, v_{a 20}, v_{b 10}$ and $v_{b 20}$ depend on the conduction states of the power switches, i.e.

$$
v_{j 0}=\left(2 s_{q j}-1\right) \frac{v_{c}}{2}, \text { for } j=\mathrm{a} 1 \text { to b2 }
$$

Where $v_{c}$ is the total dc-link voltage. Considering that $v_{a}^{*}, v_{b}^{*}$ and $v_{0}^{*}$ denote the reference voltages determined by the current controllers.

$$
\begin{aligned}
& v_{a}^{*}=v_{a 10}^{*}-v_{a 20}^{*} \\
& v_{b}^{*}=v_{b 10}^{*}-v_{b 20}^{*} \\
& v_{0}^{*}=v_{a 10}^{*}+v_{a 20}^{*}-v_{b 10}^{*}-v_{b 20}^{*}
\end{aligned}
$$


The gating signals are directly calculated from the reference pole voltages $v_{a 10}^{*}, v_{a 20}^{*}, v_{b 10}^{*}$ and $v_{b 20}^{*}$. However, (23)-(25) are not sufficient to determine the four pole voltages uniquely from $v_{a}^{*}, v_{b}^{*}$ and $v_{0}^{*}$. Introducing an auxiliary variable $v_{x}^{*}=v_{a 20}^{*}$, that equation plus the three equations (23)-(25) constitute a four independent equations system with four variables $\left(v_{a 10}^{*}, v_{a 20}^{*}, v_{b 10}^{*}\right.$ and $\left.v_{b 20}^{*}\right)$. Solving this system of equations, we obtain

$$
\begin{aligned}
& v_{a 10}^{*}=v_{a}^{*}+v_{x}^{*} \\
& v_{a 20}^{*}=v_{x}^{*} \\
& v_{b 10}^{*}=\frac{v_{a}^{*}}{2}+\frac{v_{b}^{*}}{2}-\frac{v_{0}^{*}}{2}+v_{x}^{*} \\
& v_{b 20}^{*}=\frac{v_{a}^{*}}{2}-\frac{v_{b}^{*}}{2}-\frac{v_{0}^{*}}{2}+v_{x}^{*}
\end{aligned}
$$

From these equations, it can be seen that, besides $v_{a}^{*}, v_{b}^{*}$ and $v_{0}^{*}$, the pole voltages depend on also of $v_{x}^{*}$. The limit values of the variable $v_{x}^{*}$ can be calculated by taking into account the maximum $v_{c}^{*} / 2$ and minimum $-v_{c}^{*} / 2$ value of the pole voltages.

$$
\begin{aligned}
& v_{x \max }^{*}=\frac{v_{c}^{*}}{2}-v_{\text {max }}^{*} \\
& v_{x \min }^{*}=\frac{v_{c}^{*}}{2}-v_{\text {min }}^{*}
\end{aligned}
$$

Where $v_{c}^{*}$ is the reference dc-link voltages, $v_{\max }^{*}=\max \vartheta$ and $v_{\text {min }}^{*}=\min \vartheta$, with $\vartheta=\left\{v_{a}^{*}, 0, v_{a}^{*} / 2+v_{b}^{*} / 2-\right.$ $\left.v_{0}^{*} / 2, v_{a}^{*} / 2-v_{b}^{*} / 2-v_{0}^{*} / 2\right\}$.

Introducing a parameter $\mu(0 \leq \mu \leq 1)$, the variable $v_{x}^{*}$ can be written as

$$
v_{x}^{*}=\mu v_{x \max }^{*}+(1-\mu) v_{x \min }^{*}
$$

When $\mu=0, \mu=0.5$, and $\mu=1$ the auxiliary variable $v_{x}^{*}$ Has the following values $v_{x}^{*}=v_{x \min }^{*}, v_{x}^{*}=v_{x a v e}^{*}=\left(v_{x \min }^{*}\right.$ $\left.+v_{x \max }^{*}\right) / 2$ and $v_{x}^{*}=v_{x \max }^{*}$, respectively. When $v_{x}^{*}=v_{x \max }^{*}$ or $v_{x}^{*}=v_{x \min }^{*}$ a converter leg operates with zero switching frequency. The gating signals are obtained by comparing pole voltages with one $\left(v_{t 1}\right)$, two $\left(v_{t 1}\right.$ and $\left.v_{t 2}\right)$ or more highfrequency triangular carrier signals. In the case of doublecarrier approach, the phase shift of the two triangular carrier signals $\left(v_{t 1}\right.$ and $\left.v_{t 2}\right)$ is $180^{\circ}$. The parameter $\mu$ changes the place of the voltage pulses related to $v_{a}$ and $v_{b}$. When $v_{x}^{*}=v_{x \min }^{*}(\mu$ $=0)$ or $v_{x}^{*}=v_{x \max }^{*}(\mu=1)$ are selected, the pulses are placed in the begin or in the end of the half period (Ts) of the triangular carrier signal On the other hand, when $v_{x}^{*}=v_{x a v e}^{*}$ the pulsesare centered in the half period of the carrier signal.

The change of the position of the voltage pulses leads also to the change in the distribution of the zero instantaneous voltages (i.e., $v_{a}=0$ and $v_{b}=0$ ). With $\mu=0$ or $\mu=1$ the zero instantaneous voltages are placed at the beginning or at the end of the switching period, respectively, while with $\mu=0.5$, they are distributed equally at the beginning and at the end of the half period. This is similar to the distribution of the zerovoltage vector in the three-phase inverter.

\subsection{Control Strategy}

Fig.13 presents the control block diagram of the system in Fig. 12. highlighting the control of the rectifier, to control the dclink voltage and to guarantee the grid power factor close to one. Additionally, the circulating current $i_{0}$ in the rectifier of the proposed system needs to be controlled.

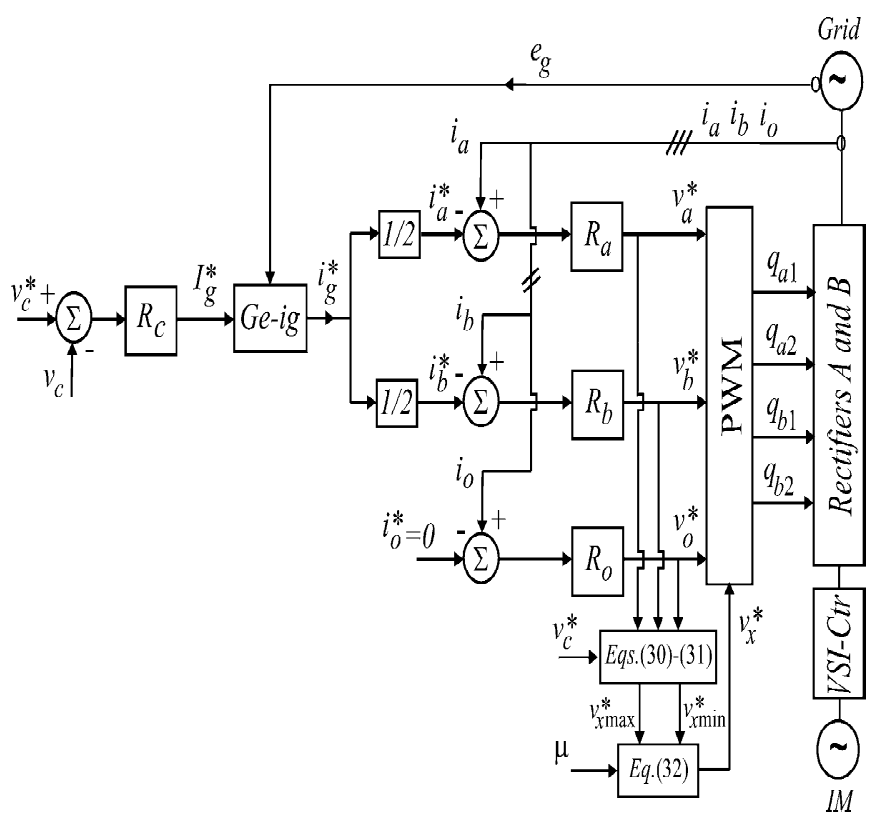

Fig.13 Control block diagram

In this way, the dc-link voltage $v_{c}$ is adjusted to its reference value $v_{c}^{*}$ using the controller $R_{c}$. which is standard Fuzzy logic controllers. This controller provides the amplitude of the reference grid current $i_{g}^{*}$ To control power factor and harmonics in the grid side, the instantaneous reference current $i_{g}^{*}$ must be synchronized with voltage $e_{g}$, as given in the voltage-oriented control (VOC) for three-phase system This is obtained via blocks $G e-i_{g}$, based on a PLL scheme. The reference currents $i_{a}^{*}$ and $i_{b}^{*}$ are obtained by making $i_{a}^{*}=i_{b}^{*}=i_{g}^{*} / 2$. Which means that each rectifier receives half of the grid current. The control of the rectifier currents is implemented using the controllers indicated by blocks $R_{a}$ and $R_{b}$. These controllers can be implemented using linear or nonlinear techniques.

The homo polar current is measured $\left(i_{0}\right)$ and compared to its reference $\left(i_{0}^{*}=0\right)$. The error is the input of Fuzzy controller $R_{0}$ that determines the voltage $v_{0}^{*}$. The calculation of voltage $v_{x}^{*}$ isgiven from (30) to (32) as a function of $\mu$, selected. The 
motor there phase voltages are supplied from the inverter (VSI).

Block VSI-Ctr indicates the inverter and its control. The control system is composed of the PWM command and a torque or flux control strategy.

\subsection{Harmonic Distortion}

The harmonic distortion of the converter voltages has been evaluated by using the weighted THD (WTHD). It is computed by using

$$
W T H D(p)=\frac{100}{a_{1}} \sqrt{\sum_{i=2}^{p}\left(\frac{a_{i}}{i}\right)^{2}}
$$

Where $a_{1}$ the amplitude of the fundamental voltage is is $a_{i}$ is the amplitude of $i$ th harmonic and $p$ is the number of harmonics taken into consideration. Fig.5.4. shows the WTHD of voltages generated by rectifiers $v_{a b}=\left(v_{a}+v_{b}\right) / 2$ for the proposed configuration and $v_{g}=v_{g 10}-v_{g 20}$ for the conventional one] at rated grid voltage asa function of $\mu$. Note that the parameter $\mu$ determines $v_{x}^{*}$ from (30) to (32). The resultant voltage $v_{a b}$ generated by rectifieris responsible to control $i_{g}$, which means that this voltage is used to regulate the harmonic distortion of the utility grid.

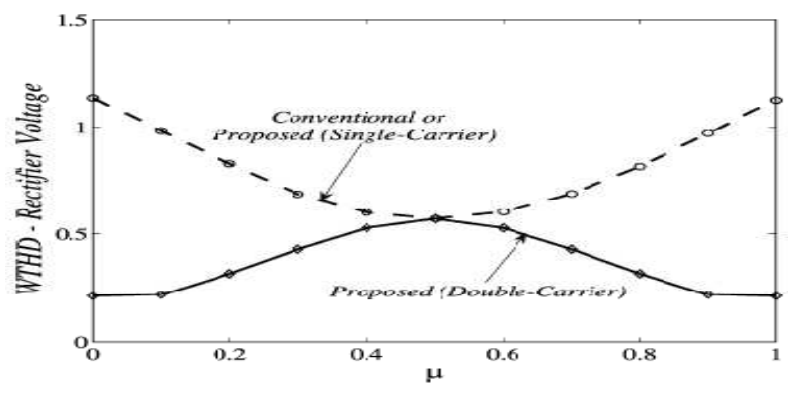

Fig 14 WTHD of rectifier voltage as a function of $\mu$.

When the single-carrier PWM is used, the behavior of WTHD of the proposed system is similar to that of conventional one for all $\mu$, as observed in Fig.5.4. When the double-carrier PWM is used with $\mu=0.5$, the WTHD is also the same for both configurations. However, for the other values of $\mu$ the WTHD of the proposed system is lower than that of the conventional one.

The WTHD of the proposed topology (double-carrier with $\mu=$ 0or $\mu=1$ ) is close to $63 \%$ of that of the conventional topology(with $\mu=0.5$ ). The study has also shown that it is possible to reduce the switching frequency of the proposed system in $60 \%$ and still have the same WTHD of the standard configuration. (a)

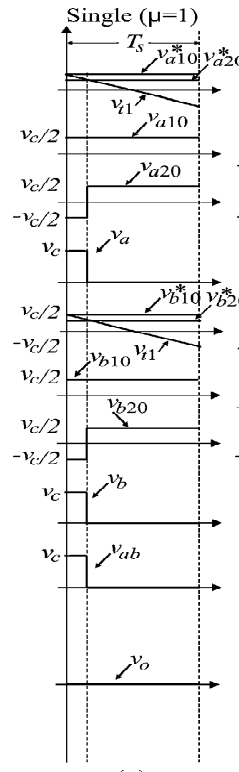

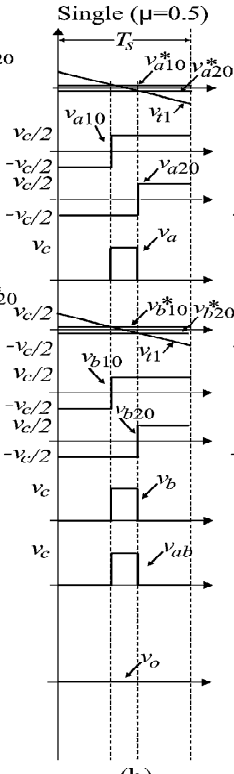

(b)

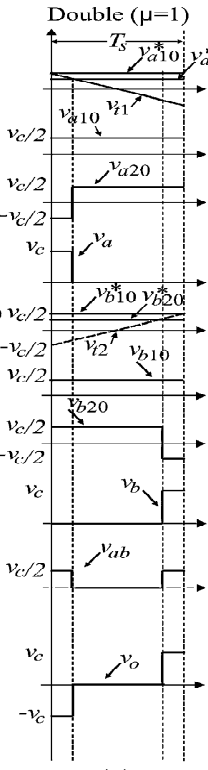

(c)

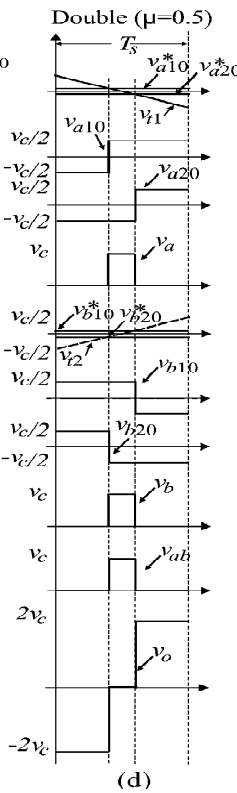

(d)
Fig. 15 Variables of rectifiers A and B. (a) Single-carrier with $\mu=1$.

(b) Single-carrier with $\mu=0.5$. (c) Double-carrier with $\mu=1$.

(d) Double carrier with $\mu=0.5$.

The WTHD behavior in Fig. 14 can be explained from Fig. 15.That figure depicts the pole voltages $\left(v_{a 10}, v_{a 20}, v_{b 10}, v_{b 20}\right)$ and their references $\left(v_{a 10}^{*}, v_{a 20}^{*}, v_{b 10}^{*}, v_{b 20}^{*}\right)$, the triangular carrier signals $\left(v_{t 1}, v_{t 2}\right)$, the resultant rectifier voltage $\left(v_{a b}\right)$ and the circulating voltage $\left(v_{0}\right)$. Fig 15 (a) and (c) shows these variables with single-carrier (with $\mu=1$ ) and double-carrier (with $\mu=1$ ), respectively.

For the double-carrier see Fig. 15 (c) the voltage $v_{a b}$ has smaller amplitude and better distribution along the half switching period than that of single-carrier see Fig. 15 (a),which means a lower $W T H D$ (as observed in Fig. 4 for $\mu=$ $1)$.

On the other hand, for $\mu=0.5$ see Fig. 15 (b) and (d) the distribution of voltage $v_{a b}$ along the switching period is the same for both cases, i.e., single-carrier and double-carrier have the same WTHD. Besides the total harmonic distortion (THD) of the grid current $i_{g}$, associated to the WTHD of the voltage $v_{a b}$, the harmonic distortion analysis must also consider the currents in the rectifiers. This is an important issue due to losses of the converter.

The harmonic distortion of the rectifier current $\left(i_{a}, i_{a}^{\prime}, i_{b}, i_{b}^{\prime}\right)$ with double-carrier is higher than that of the grid current $i g$. When the parallel rectifier with double-carrier is used, the $T H D$ of all these currents are reduced for $\mu=0$ or $\mu=1$ and increased for $\mu=0.5$. On the other hand, the THD of the circulating current is also smaller with $\mu=0$ or $\mu=1$. Fig. 16 
shows currents $i_{a}, i_{a}^{\prime}$, and $i_{0}$ for double-carrier with $\mu=1$ and $\mu=0.5$. It can be seen that the mean values of the ripples of all currents are smaller when $\mu=1$ is selected..
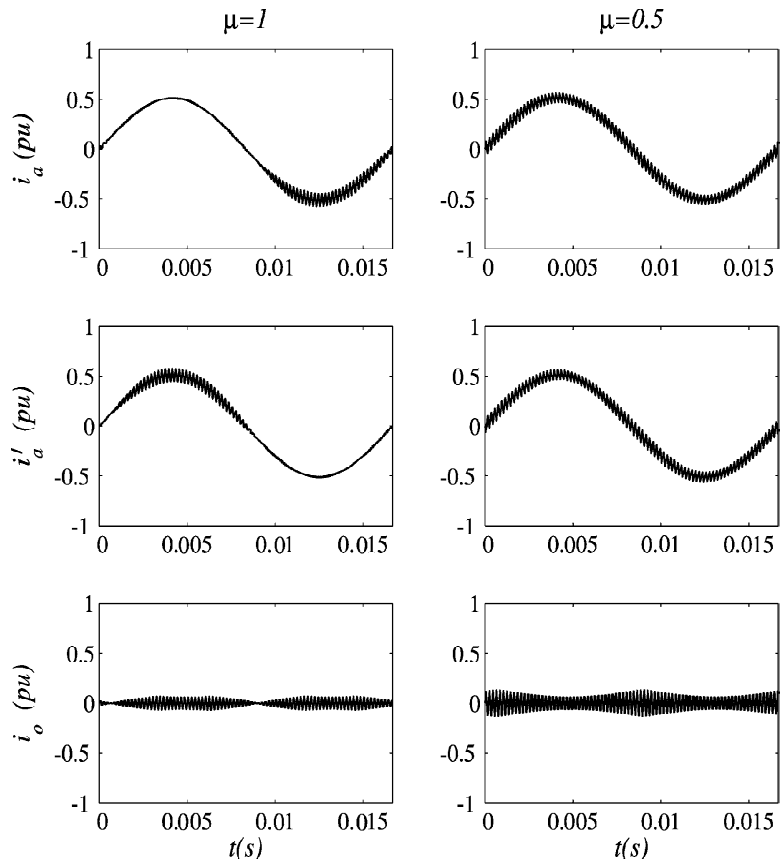

Fig 16 currents $i_{a}, i_{a}^{\prime}$, and $i_{0}$ for double-carrier with $\mu$ $=1$ and $\mu=0.5$

In conclusion the optimal rectifier operation is obtained with double-carrier making $\mu=0$ or $\mu=1$. A four-carrier approach may also be used.

\subsection{Ratings of Switches}

Assuming same rms voltages at both grid and machine sides ,a machine power factor of 0.85 and neglecting the converter losses, currents of the rectifier switches normalized in terms of currents of the inverter switches are 2.55 and 1.27 for the conventional and the proposed single-phase to three-phase converter, respectively. Fig (a) and (b) shows the flow of active power in the conventional and in the proposed singlephase to three-phase converter, respectively. For balanced system $\left(L_{g}^{\prime}=L_{a}=L_{a}^{\prime}=L_{b}=L_{b}^{\prime}\right)$, voltage $v_{0}$ is close to zero, so that the dc-link voltage is equal to that required by the conventional system. Since the parallel connection scheme permits to reduce the switch currents and preserve the dc-link voltage, the rating of each power switch in the rectifier side is reduced.

\section{SIMULATION MODELING AND RESULTS}

\subsection{Simulation Modeling for Transient Condition}

In this chapter the work carried out on mathematical model of the system using MATLAB/SIMULINK software. The results of simulation have been discussed.

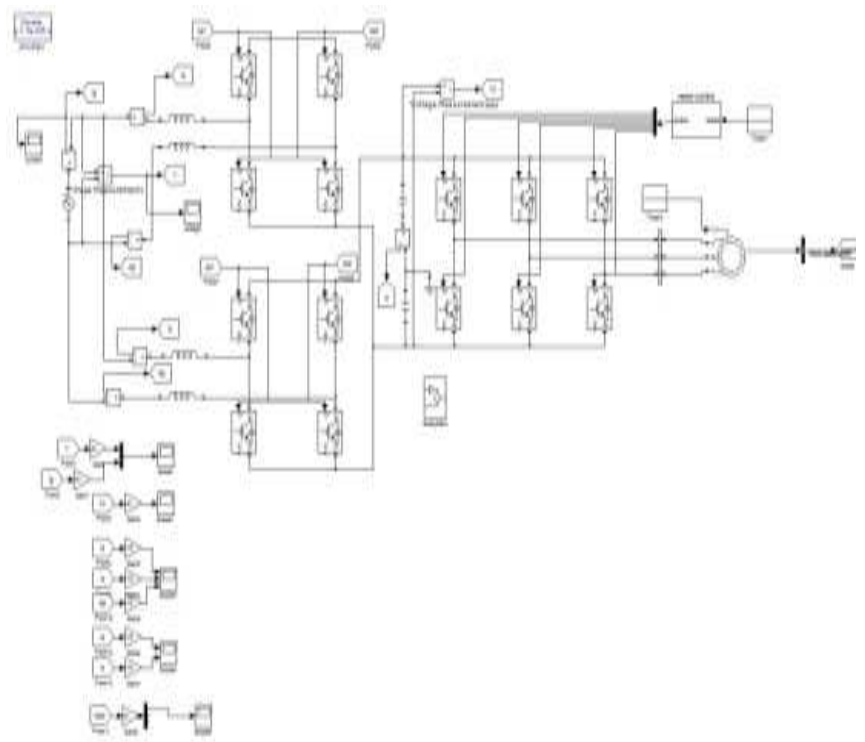

Fig 17 Simulation model of single phase to three phase drive system using two parallel single phase rectifiers

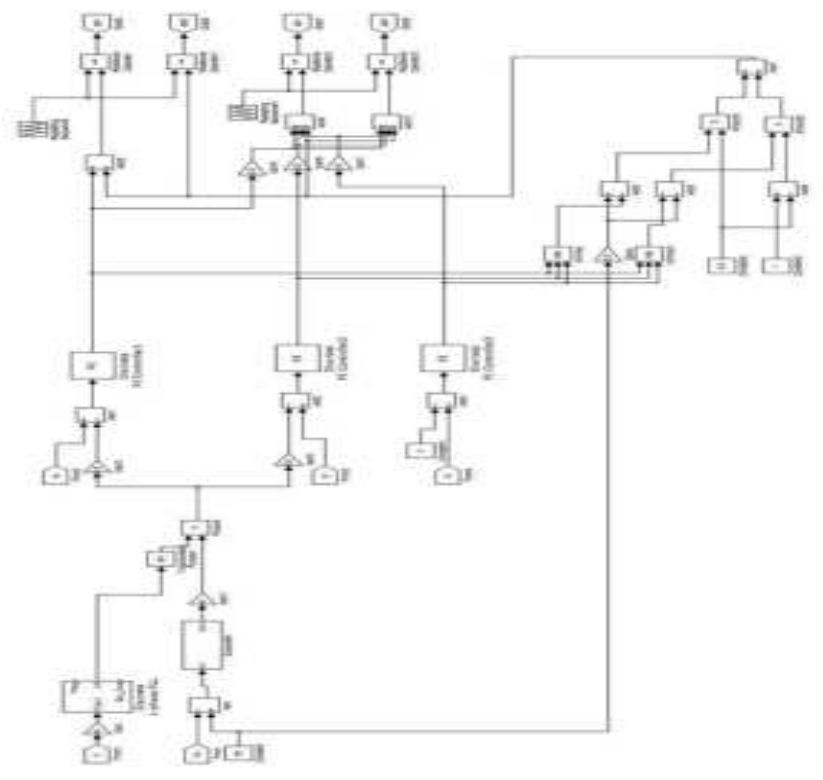

Fig 18 (a) Control Block Diagram 


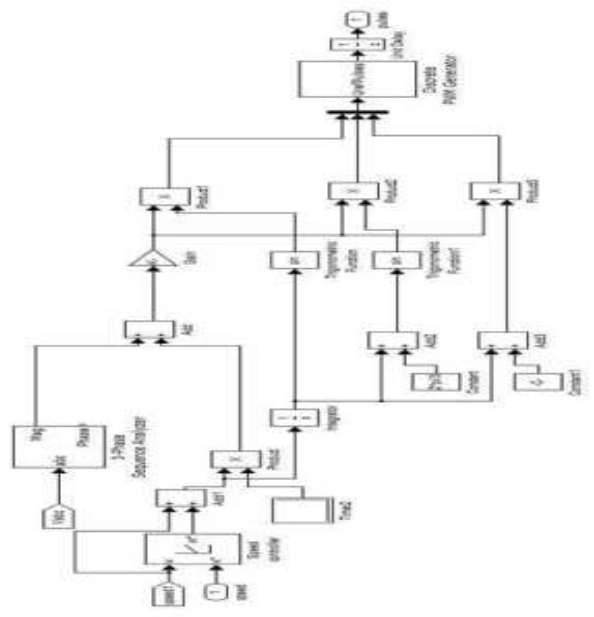

Fig 18 (b) Space Vector PWM Diagram

\subsection{Simulation Results for Transient Condition}

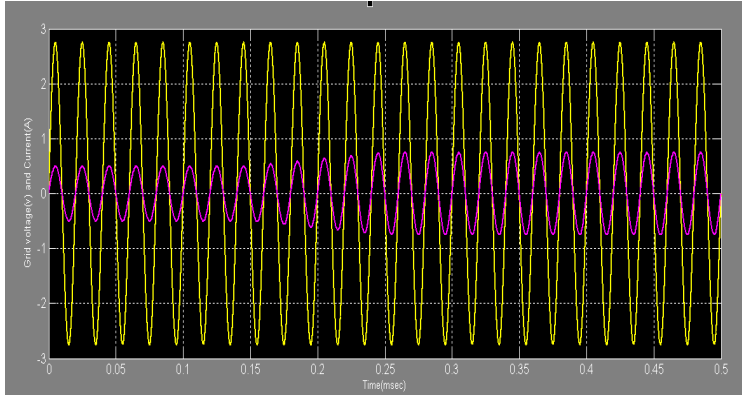

Fig 19(a) Grid voltage and current

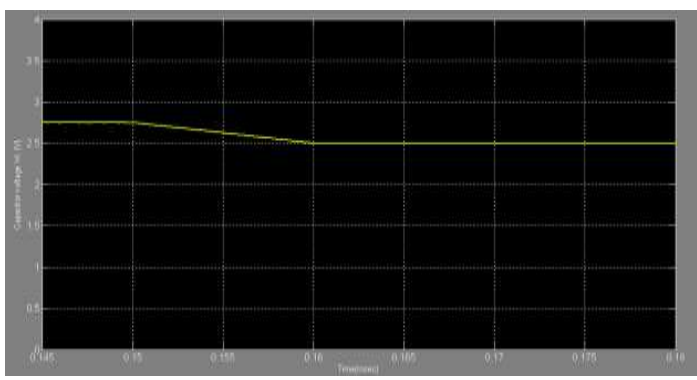

Fig 19(b) Capacitor voltage

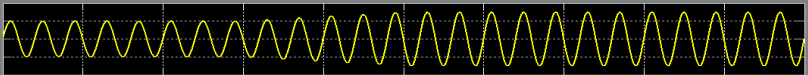

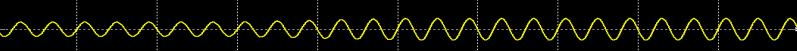

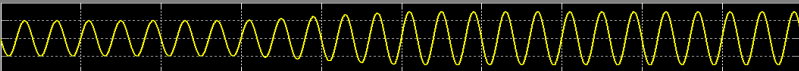

Fig 19(c) Currents of rectifier A ( ia and ia') and circulating current

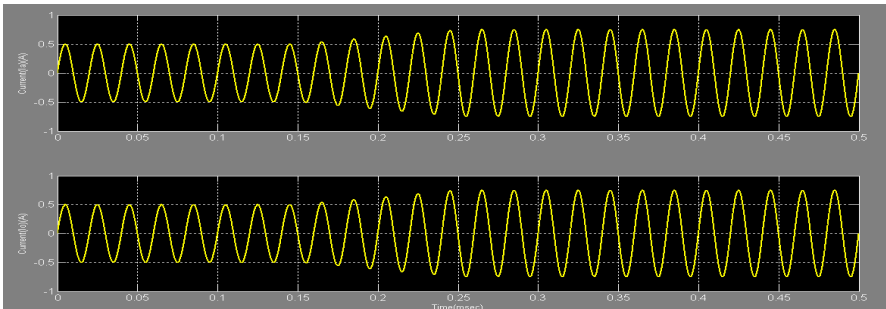

Fig 19(d) Currents of rectifier A (ia) and rectifier B current (ib)

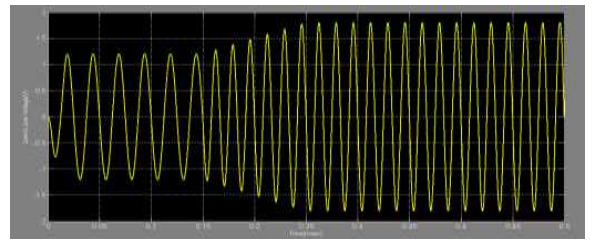

Fig 19(e) Line to line voltage of load

6.3 Simulation Modeling Faulty Zone at Rectifier

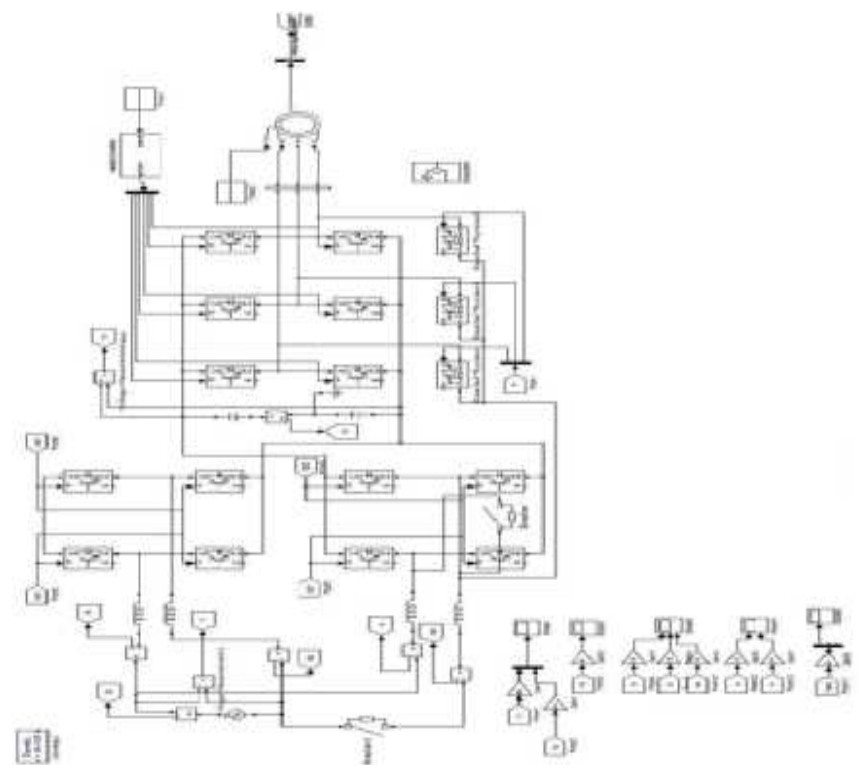

Fig20 Fault Identified at rectifier B

6.3.1 Simulation results for fault at rectifier $B$

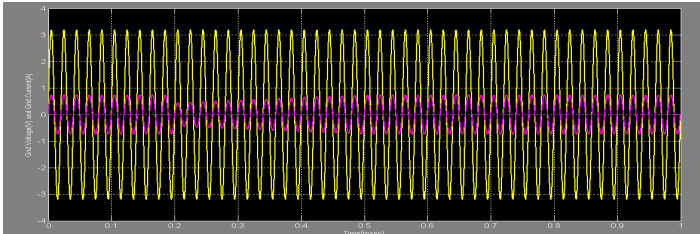


Fig 21(a) Grid voltage and grid current

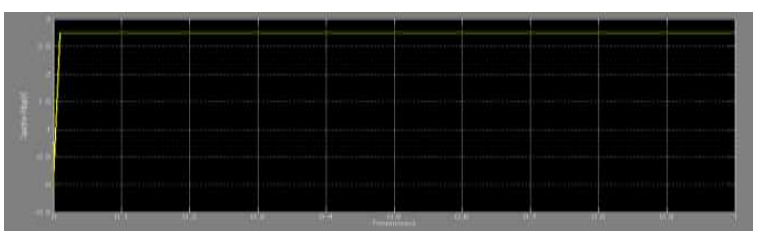

Fig 21(b) DC link capacitor voltage

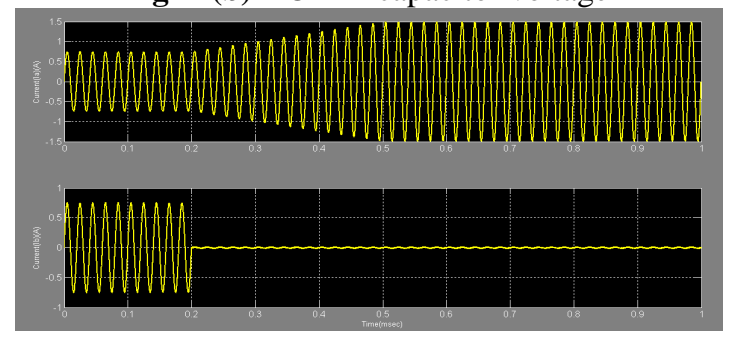

Fig 21(c) Currents of rectifier A and B

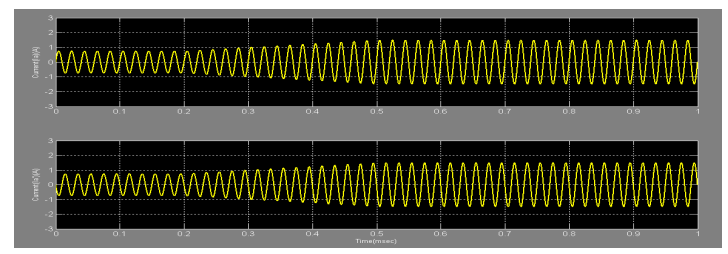

Fig 21 (d) Currents of rectifier (ia and ia')

\subsubsection{Output Wave Form Explanation for Transient}

\section{Condition.}

1. Figure 19(a) represents grid voltage and current at transient condition. grid voltage is $110 \mathrm{~V}$,grid current is $8 \mathrm{~A}$. At the transient condition grid current having some distortions, after some it will reaches to steady state condition. Time taken for steady state is $0.25 \mathrm{~m}$ sec.

2. Figure 19(b) represents the DC link Capacitor voltage. At steady state capacitor value is $100 \mathrm{~V}$.

3. Figure 19(c) represents rectifier $A$ currents $i_{a}$ and $i_{a}$ '. The current shared by rectifier A is $4 \mathrm{~A}$. it is the half of grid current. Circulating current is represented.

4. Figure 19(d) represents rectifier A current and rectifier $\mathrm{B}$ currents at transient condition. When it reaches to steady state value the currents of rectifier $\mathrm{A}$ and $\mathrm{B}$ is $4 \mathrm{~A}$.

5. Figure 19(e) represents line to line voltage of load. At transient condition it is low value. At steady state line voltage of load is $125 \mathrm{~V}$.

\subsubsection{Output Wave Form Explanation for Fault At}

\section{Rectifier B}

1. Figure 21(a) represents grid voltage and currents. From the figure up to $0.2 \mathrm{~m} \mathrm{sec}$ rectifier A and B is in healthy condition then current sharing is equal. When fault occurred in rectifier $\mathrm{B}$ the total grid current $8 \mathrm{~A}$ is shared by Rectifier A.

2. Figure 21(b) represents the capacitor voltage. The capacitor voltage $100 \mathrm{~V}$. when fault occurred rectifier $\mathrm{B}$ capacitor voltage decreases and again it reaches to $100 \mathrm{~V}$.

3. Figure 21(c) represents the rectifiers currents when fault at rectifier $\mathrm{B}$. The total grid current is shared by rectifier A is started at $0.5 \mathrm{~m} \mathrm{sec}$.

4. Figure 21(d) represents rectifier currents ia and ia'. The current magnitude of these two currents is

\section{CONCLUSIONS}

A single-phase to three-phase drive system composed of two parallel single-phase rectifiers, a three-phase inverter and an induction motor was proposed. The system combines two parallel rectifiers without the use of transformers. The system model and the control strategy, including the PWM technique, have been developed.

The complete comparison between the proposed and standard configurations has been carried out in this paper. Compared to the conventional topology, the proposed system permits to reduce the rectifier switch currents, the THD of the grid current with same switching frequency or the switching frequency with same $T H D$ of the grid current and to increase the fault tolerance characteristics. In addition, the losses of the proposed system may be lower than that of the conventional counterpart.

The initial investment of the proposed system (due to high number of semiconductor devices) cannot be considered a drawback, especially considering the scenario where the cited advantages justify such initial investment. The experimental results have shown that the system is controlled properly, even with transient and occurrence of fault

\section{FUTURE SCOPE}

It is quite common to have only a single phase power grid in residential, commercial, manufacturing, and mainly in rural areas. At the solar power plant and industries it is convenient convert single phase supply to three phase supply. This system is very useful for agricultural to run three phase motor.

\section{REFERENCES}

[1] D.-C. Lee and Y.-S. Kim, "Control of single-phase-tothree-phase AC/DC/AC PWM converters for induction 
motor drives," IEEE Trans. Ind. Electron., vol. 54, no. 2, pp. 797-804, Apr. 2007.

[2] D. J. Nowak, A. Bendre, G. Oriti, and A. L. Julian, "Mitigating circulating common-mode currents between parallel soft-switched drive systems," IEEE Trans. Ind. Appl., vol. 43, no. 5, pp. 1284-1294,. R. M. Cuzner, Sep./Oct. 2007.

[3] C. B. Jacobina, E. C. dos Santos Jr., E. R. C. da Silva, M. B. R. Correa, A. M. N. Lima, and T. M. Oliveira, "Reduced switch count multiple three phase ac machine drive systems," IEEE Trans. Power Electron., vol. 23, no. 2, pp. 966-976,

[4] Z. Ye, P. Jain, and P. Sen, "Circulating current minimization in high frequency AC power distribution architecture with multiple inverter modules operated in parallel," IEEE Trans. Ind. Electron., vol. 54, no. 5, pp. 2673-2687, Oct. 2007.

[5] P.-T. Cheng, C.-C. Hou, and J.-S. Li, "Design of an auxiliary converter for the diode rectifier and the analysis of the circulating current," IEEE Trans. Power Electron., vol. 23, no. 4, pp. 1658-1667, Jul. 2008.

[6] H. Cai, R. Zhao, and H. Yang, "Study on ideal operation status of parallel inverters," IEEE Trans. Power Electron., vol. 23, no. 6, pp. 2964-2969, Nov. 2008.

[7] J. Holtz, "Pulse width modulation for electronic power conversion," Proc. 